\title{
Morphology-Based Crack Detection for Steel Slabs
}

\author{
Anders Landström and Matthew J. Thurley
}

\begin{abstract}
Continuous casting is a highly efficient process used to produce most of the world steel production tonnage, but can cause cracks in the semi-finished steel product output. These cracks may cause problems further down the production chain, and detecting them early in the process would avoid unnecessary and costly processing of the defective goods. In order for a crack detection system to be accepted in industry, however, false detection of cracks in non-defective goods must be avoided. This is further complicated by the presence of scales; a brittle, often cracked, top layer originating from the casting process.

We present an approach for an automated on-line crack detection system, based on 3D profile data of steel slab surfaces, utilizing morphological image processing and statistical classification by logistic regression. The initial segmentation successfully extracts $80 \%$ of the crack length present in the data, while discarding most potential pseudo-defects (non-defect surface features similar to defects). The subsequent statistical classification individually has a crack detection accuracy of over $80 \%$ (with respect to total segmented crack length), while discarding all remaining manually identified pseudo-defects. Taking more ambiguous regions into account gives a worst-case false classification of $131 \mathrm{~mm}$ within the $30600 \mathrm{~mm}$ long sequence of $150 \mathrm{~mm}$ wide regions used as validation data. The combined system successfully identifies over $70 \%$ of the manually identified (unambiguous) crack length, while missing only a few crack regions containing short crack segments.

The results provide proof-of-concept for a fully automated crack detection system based on the presented method.
\end{abstract}

Index Terms-Mathematical morphology, crack detection, steel slabs.

\section{INTRODUCTION}

\section{A. Background}

Currently almost $95 \%$ of the world steel production tonnage is solidified by continuous casting [1]. The method has advantages in productivity, cost reduction and output quality, but, depending on factors such as design, operation and maintenance, may introduce various surface defects [2]. Steel slabs (see fig. 1), the major (semi-finished) product within steel casting, are therefore susceptible to crack formation.

Since steel slabs are often intended for sheet steel rolling, surface defects such as cracks may result in long sections of defective end-user products. Consequently, inspection of steel slabs before sending them through to the rolling mill, thereby avoiding related problems at the later stage, is important to the steel industry. However, most inspection systems within the area are still manually operated [3], [4].

This work focuses on automated detection of longitudinal cracks in steel slabs, based on non-contact measurements. A good solution to the problem should efficiently detect

A. Landström and M.J. Thurley are with the Department of Computer Science, Electrical and Space Engineering, Luleå University of Technology, Luleå, Sweden.

Manuscript received February 6, 2012; revised May 30, 2012. potentially problematic cracks, while keeping the number of false positives (non-crack regions being identified as cracks) at a minimum. Therefore particular notice needs to be taken to the presence of scales, which constitute a brittle, often cracked, top layer, formed from oxidization in the manufacturing process. This scale layer is unavoidable during casting [5], and cracks therein are from a top view perspective similar to cracks in the steel and therefore risk causing false positives in the detection result. In the intended use of this work, a robust system must handle surfaces partially covered by scales without reporting false positives from pseudo-defects (nondefect surface features similar to defects).

\section{B. Related Research}

Surface inspection by computer vision is a wide topic, and its progress in industrial processes has been documented in several surveys during the last decades [6]-[11].

Of particular interest to this work are automated systems for steel surface inspection, which is a field of ongoing study well represented in literature. Gray-scale intensity imaging is commonly used, in combination with various different signal processing techniques such as wavelet transforms [12], [13], Gabor filters [4] and image morphology [4], [5], [14]. Use of gray-scale intensity images has its limitations though. Variations in lightning conditions, giving rise to potential pseudo-defects, are a problem. In particular, light reflection from scale regions may vary substantially, making the gray level in intensity images highly unpredictable which may give rise to psuedo-defects [13]. Other parameters, such as steel type, may effect properties in gray-scale images as well [5].

Due to the shortcomings of intensity imaging, other alternative optical techniques for automated inspection of metallic surfaces have been suggested. Pernkopf [15] notes that range imaging provides better contrast to surface defects with "threedimensional characteristics", and that the strong changes in reflective properties in scale regions motivates the use of range

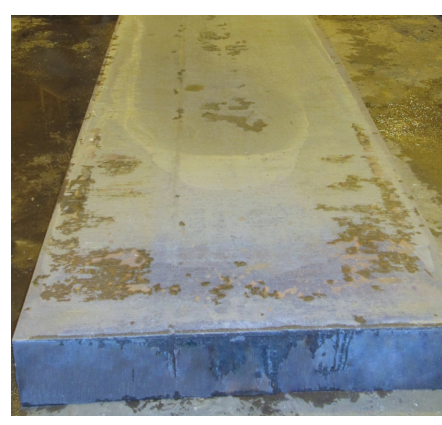

(a)

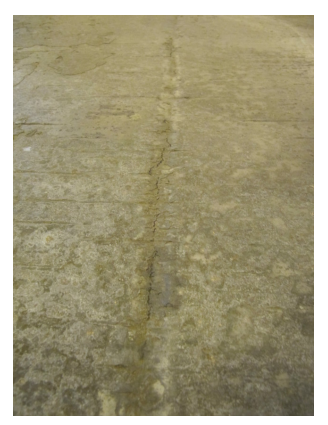

(b)
Fig. 1. Examples of a steel slab (a) and a longitudinal surface crack (b). 
data over intensity imaging due to less sensitivity to inhomogeneous reflectance. Pernkopf and O'Leary [16] summarize two range imaging methods: Light sectioning, using projected light to calculate distance, and photometric stereo, obtaining distances for a static scene from several intensity images using different light sources. Another solution, based on range data collected by conoscopic holography, is presented by Alvarez et al. [17].

In addition to the more traditional machine vision approaches, numerous systems interpreting data collected by other Non-Destructive Testing (NDT) methods such as thermocouples [18], eddy currents [19], magnetic powder [20], ultrasound [21] or sulfur prints [22] are also common in literature. These systems generally require more contact though, in contrast to intensity and range imaging.

In addition to the given examples of methods for steel inspection, other methods for crack detection have been applied in other contexts. The possibility to use watershed segmentation for crack detection in X-ray images of welds have been studied [23], and the Hough transform have been used for crack detection in color images of biscuits [24] as well as X-ray images of welds [25].

Watershed segmentation can be used to identify cracks, given a proper set of seeds, but risks over-segmenting the data [23]. The method may therefore require substantial postprocessing in order to properly separate cracks from pseudodefects. A good set of seeds reduces post-processing, but instead increases the need for pre-processing. For example, use of stochastic watershed [26] would require a minimum amount of parameters while providing a good segmentation, but requires a substantial amount of processing time and is therefore not suitable for the intended on-line system. Moreover, when no crack is present watershed segmentation will still produce something which must be post-processed in order to rule out the presence of cracks.

The Hough transform is a good tool for identifying line segments in the data, and have been used in crack detection systems [24], [25]. However, the Hough transform requires a binary image as input, which again requires pre-processing. The situation is also complicated by the fact that cracks in steel may not be perfectly straight, which further calls for pre-processing.

\section{Contribution}

As stated in the previous section, inspection of metallic surfaces by intensity imaging suffers from limitations. In particular, the method may introduce pseudo-defects in scale regions. For robust non-contact inspection of steel surfaces partially covered by scales, range imaging provides a more promising alternative.

Due to the nature of the presented problem, where the cracks by definition have a longitudinal orientation and thereby represent a specific directional structure in the casted steel surface data, we present a solution based on mathematical morphology. The strength of mathematical morphology lies in its ability to identify and/or enhance features of specific shape and orientation in the data. It is a common technique for analyzing

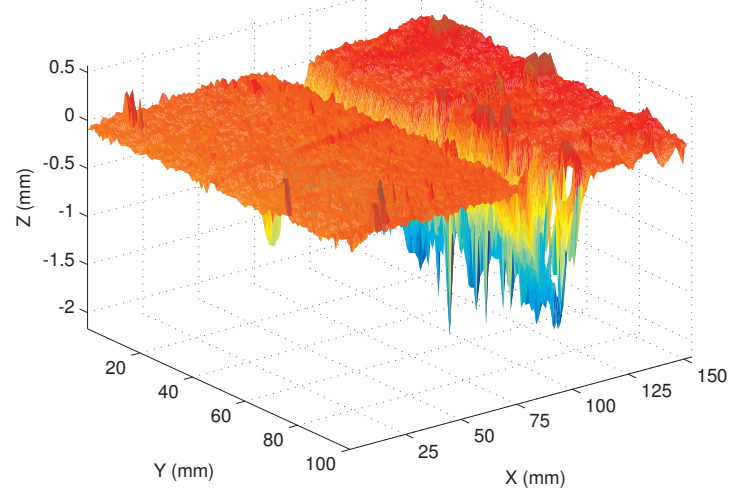

(a)

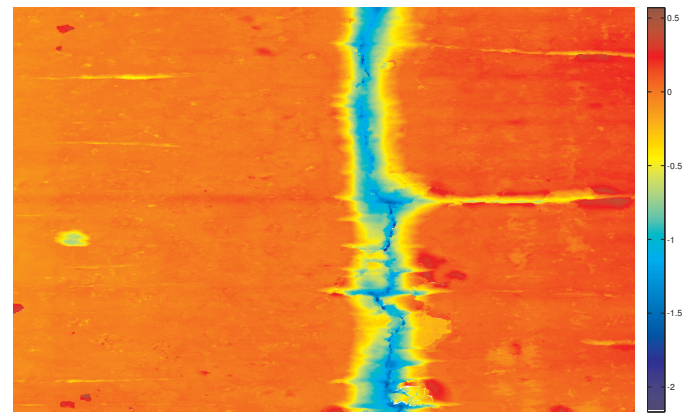

(b)

Fig. 2. Input data example: A $150 \times 100 \mathrm{~mm}$ (width $\times$ length) region of steel slab profile data, viewed in 3D (a) and from above (b).

3D profile data, and has previously been used in a wide range of applications such as LiDAR [27], aggregates [28], [29], and pellets [30]. However, it has to the authors' knowledge not been used for analysis of 3D profile data in the intended context; crack detection for casted steel.

In this work, we present a strategy for morphology-based crack detection for steel slabs based on 3D surface profile data collected by laser triangulation. The system first segments the data using mathematical morphology, and the resulting connected regions are assigned a crack probability using a logistic regression model.

\section{Measurements}

Sets of 3D profile data for steel slab surfaces were acquired at two separate occasions, and are hence referred to as sets A and $\mathrm{B}$, respectively. The data is processed in regions (here referred to as images) of $150 \times 100 \mathrm{~mm}$ (width $\times$ length, or $\mathrm{x} \times \mathrm{y}$ ) in size, identifying cracks by segmenting the data set and classifying the resulting connected regions. Set A, containing a total of 644 images collected from two slabs, was used as model set for the presented segmentation algorithm and thereafter used to train a classifier, while set $\mathrm{B}$, containing a total of 323 images collected from four slabs, was used as an independent validation set. Reference maps for both sets, defining the location of strong crack signatures in the data, were produced by manually marking clear, open cracks in the data. Fig. 2 presents an example of crack data, captured at $0.1 \times 0.1 \times 0.0053 \mathrm{~mm}($ width $\times$ length $\times$ depth $)$ resolution. 
TABLE I

MORPHOLOGICAL NOTATION

\begin{tabular}{l|l}
$B$ & Binary image. \\
$I$ & Gray-scale image. \\
$s$ & Structuring element. \\
$I \ominus s$ & Erosion of $I$ by $s$. \\
$I \oplus s$ & Dilation of $I$ by $s$. \\
$I \circ s=(I \ominus s) \oplus s$ & Opening of $I$ by $s$. \\
$I \bullet s=(I \oplus s) \ominus s$ & Closing of $I$ by $s$. \\
$I r e c \oplus 8$ & 8-pixel neighborhood reconstruction \\
& of $I$ from a marker image $I_{M}$.
\end{tabular}

\section{Segmentation}

Morphological image processing is frequently used throughout this section. Definitions of the morphological concepts can be found in the introduction to the subject provided by Dougherty and Lotufo [31], and notations used are summarized in Table I. The nature of the morphological operations give rise to a set of parameters, which must be set in accordance to a definition of what we are actually looking for. Therefore these parameters have been set experimentally from the model set, while considering their relation to adequate physical measures relevant to the problem. We thereby define the following concepts:

- Trench: A single-directional, less than $5 \mathrm{~mm}$ wide, distinct longitudinal depression in the surface.

- Scale: An elevated, less than $25 \mathrm{~mm}$ wide, part of the surface, possibly cracked by an opening no more than $5 \mathrm{~mm}$ in width.

- Crack signature: A trail of the bottom $0.1 \mathrm{~mm}$ wide portions of a trench, where the trench depth is larger than $0.1 \mathrm{~mm}$.

To emphasize the relation to the physical measures, lengths are denoted in $[\mathrm{mm}]$ rather than pixels. These parameters are likely dependent of the steel grade being inspected, but an investigation of such relations lies beyond the scope of this work.

After preprocessing (described in section III-A), a coarse search for trenches is performed at $1 \times 1 \mathrm{~mm}$ resolution (section III-B). The search is then gradually refined, first at $0.3 \times 0.3 \mathrm{~mm}$ resolution where scales are excluded (section III-C) and finally at $0.1 \times 0.1$ resolution where cracks are identified (section III-D). This strategy decreases processing time and concentrates on regions where cracks are expected.

Binary images displayed in this section are inverted, displaying pixels with value 1 (true) in black on a white background.

\section{A. Preprocessing}

Before crack signatures are extracted from the data, preprocessing is performed.

1) Identifying the slab region: The actual slab region in the data is identified so that we do not search for cracks outside the slab.

2) Compensating for slope: The slope of the slab is compensated for by subtracting a least-squares fitted plane from the data, using a uniformly randomly distributed set of 15000 sample points ( $1 \%$ of the pixels) for the least-squares fit. We here assume that each $150 \times 100 \mathrm{~mm}$ part of the slab surface can be approximated by a plane.

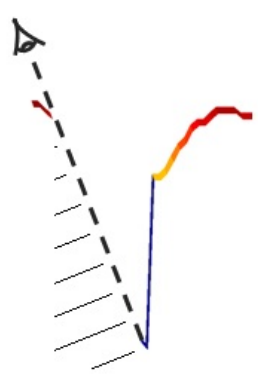

(a)

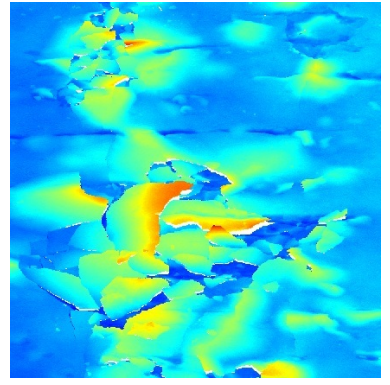

(b)
Fig. 3. (a) Side view example of occlusion: Surface information from the crossed-out region cannot reach the sensor, resulting in occluded (unknown) $3 \mathrm{D}$ profile data such as the white pixels around the scales shown in (b).

3) Handling occluded data: Occluded regions in the measured data, caused by other parts of the surface blocking the path between the projected laser line and the sensor (see fig. 3), are potential crack indicators and therefore set to the minimum height value of the currently processed $150 \times 100 \mathrm{~mm}$ region.

4) Removing noise: Noise in the data is reduced by median filtering, using a centered neighborhood of size $0.3 \times 0.3 \mathrm{~mm}$.

\section{B. Trench Search (Low Resolution)}

In order to reduce the total required processing time, an initial low resolution search for surface cavities is performed on a downsampled version of the preprocessed data set (see fig. 4a). In the downsampling process, the data is converted to $1 \times 1 \mathrm{~mm}$ resolution using a Gaussian filter before sampling (to be fully accurate the new scale is $1.1 \times 1.1 \mathrm{~mm}$ in order to use an odd sized neighborhood, but for simplicity we write $1 \mathrm{~mm}$ in this text). This resolution is too low to capture a crack in detail, but the longitudinal trench in the data indicating a potential crack is clearly visible. Such trenches can be identified by morphological processing, so that only regions in their proximity need to be processed further.

1) Estimating trench depth: Let $I_{1}$ denote the downsampled preprocessed 3D data (fig. $4 \mathrm{a}$ ) and define $l_{\mathrm{h}, 5 \mathrm{~mm}}$ and $l_{\mathrm{v}, 5 \mathrm{~mm}}$ as a horizontal and a vertical, respectively, line structuring element of length $5 \mathrm{~mm}$. The difference between the minimum heights to which the two structuring elements can be pushed down into the preprocessed steel surface,

$$
I_{\text {raw }}=\left(I_{1} \bullet l_{\mathrm{h}, 5 \mathrm{~mm}}\right)-\left(I_{1} \bullet l_{\mathrm{v}, 5 \mathrm{~mm}}\right),
$$

then yields a first approximation $I_{\text {raw }}$ of the trench depth, as presented in fig. $4 \mathrm{~b}$. The operation separates longitudinal features from transversal, i.e. cracks from oscillation marks, by assigning them different signs (longitudinal trench depths being positive).

The raw extracted signal may be effected by oscillation marks, but a more refined trench signature,

$$
I_{\text {trench }}=I_{\text {raw }} \bullet l_{\mathrm{v}, 5 \mathrm{~mm}},
$$

can be obtained by performing another morphological closing with the $5 \mathrm{~mm}$ vertical line structuring element on the data (fig. 4c). 


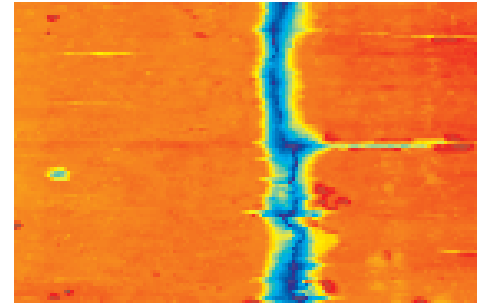

(a)

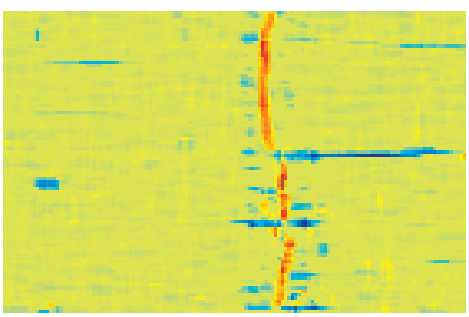

(b)

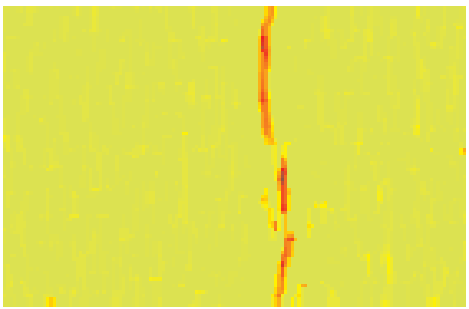

(c)

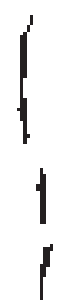

(d)

Fig. 4. The different images obtained during the trench search: $I_{1}$ (a), $I_{\text {raw }}(\mathrm{b}), I_{\text {trench }}(\mathrm{c})$, and $B_{\text {trenches }}(\mathrm{d})$.

2) Extracting trench markers: By thresholding $I_{\text {trench }}$, keeping only the top $1 \%$ largest positive trench depths, a binary marker $B_{\text {top }}$ can be obtained. A final binary trench map

$$
B_{\text {trenches }}=B_{\text {top }} \operatorname{rec}_{\oplus 8}\left(B_{\text {top }} \circ l_{\mathrm{v}, 10 \mathrm{~mm}}\right),
$$

containing regions of at least $10 \mathrm{~mm}$ (longitudinal) length connected to these largest trench depths, can then be retrieved from $B_{\text {top }}$ through opening by reconstruction. The resulting binary image is presented in fig. $4 \mathrm{~d}$.

\section{Scale Exclusion (Medium Resolution)}

Scales constitute a brittle oxidized top layer covering the casted steel (see fig. 3b). This top layer is often cracked; in particular where scales have separated from the steel surface. Hence, the measured data is likely to contain pseudo-defects in the form of cracks in the scales which do not reach down into the actual steel surface below.

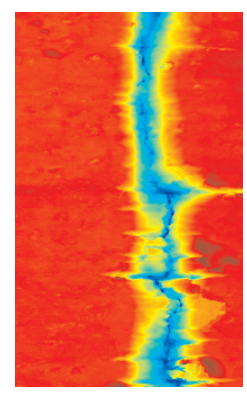

(a)

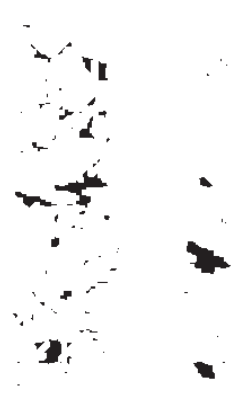

(d)

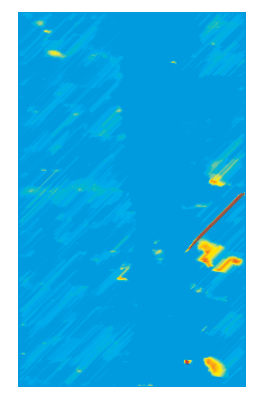

(b)

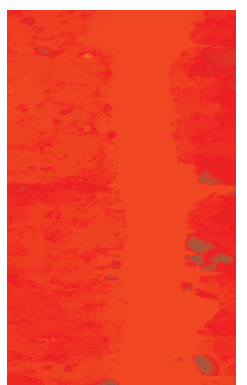

(e)

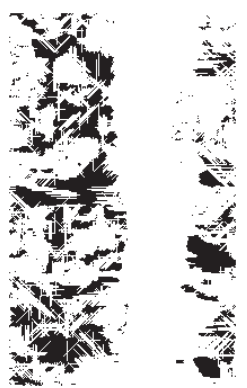

(c)

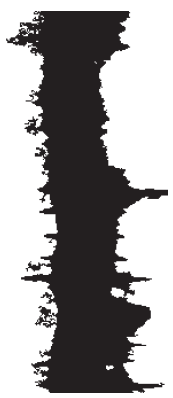

(f)
Fig. 5. The different images obtained during the scale exclusion: $I_{0.3}$ (a), $I_{\text {diff }}\left(45^{\circ}\right)(\mathrm{b}), B_{\text {scales }}(\mathrm{c}), B_{\text {scales, e }}(\mathrm{d}), I_{\text {rec }}(\mathrm{e})$, and $B_{\text {cav }}(\mathrm{f})$.

Cracks will later be identified by considering local topology variations. At that stage, a scale crack will cause a signature very similar (or even identical to) a crack in the steel. Therefore, scale regions must be excluded from further processing to avoid false crack detection. Scales do not require a $0.1 \times 0.1 \mathrm{~mm}$ resolution, and the procedure for excluding them is therefore performed at $0.3 \times 0.3 \mathrm{~mm}$ resolution in order to reduce the number of required computations.

1) Identifying potential scales in one direction: Let $I_{0.3}$ (fig. 5a) denote a downsampled region around a surface trench identified in $B_{\text {trenches }}$ (fig. $4 \mathrm{~d}$ ). Then let $l_{\theta, 5 \mathrm{~mm}}$ and $L_{\theta, 25 \mathrm{~mm}}$ denote lines of lengths $5 \mathrm{~mm}$ and $25 \mathrm{~mm}$, respectively, in the same direction $\theta$. The closing

$$
I_{\mathrm{c}}=I_{0.3} \bullet l_{\theta, 5 \mathrm{~mm}}
$$

first fills in any small gaps (less than $5 \mathrm{~mm}$ ) in the data in the direction $\theta$. A signature for (possibly cracked) potential scales less than $25 \mathrm{~mm}$ in width, as viewed from the direction $\theta$, can then be retrieved from the top-hat operation

$$
I_{\text {diff }}(\theta)=I_{\mathrm{c}}-I_{\mathrm{c}} \circ L_{\theta, 25 \mathrm{~mm}} .
$$

These identified potential scales, shown for $\theta=45^{\circ}$ in fig. $5 \mathrm{~b}$, should not correspond to crack regions due to the local surface topology.

2) Combining several directions: A single direction may give a crude result, but by combining the results from several directions this can be improved. By letting $\theta=$ $\left\{-45^{\circ}, 0^{\circ}, 45^{\circ}, 90^{\circ}\right\}$, where the angles are denoted with respect to the $\mathrm{x}$-axis (the transversal direction), a refined scale marker $B_{\text {scales }}$ (fig. $5 \mathrm{c}$ ) representing only potential scales showing up in all four angles can be retrieved from the 
expression

$$
B_{\text {scales }}=\left(\bigcap_{k=1}^{4}\left(I_{\text {diff }}\left(\theta_{k}\right)>0\right)\right) .
$$

The marker image is then eroded by a disk $d_{\mathrm{r}=1 \mathrm{~mm}}$ with radius $1 \mathrm{~mm}$,

$$
B_{\text {scales, e }}=B_{\text {scales }} \ominus d_{\mathrm{r}=1 \mathrm{~mm}},
$$

which removes noise and shrinks the sizes of the obtained marker regions. This reduces the risk of noisy markers stretching into surface cavities (compare the original marker in fig. $5 \mathrm{c}$ to the eroded marker in fig. $5 \mathrm{~d}$ ).

3) Improving markers by reconstruction: From the resulting binary marker $B_{\text {scales, e }}$ (fig. 5d), a marker $I_{\text {marker }}$ for grayscale data can be constructed by letting

$$
I_{\text {marker }}=\left\{\begin{array}{cl}
I_{0.3} & \text { where } B_{\text {scales, }}=1 \\
0 & \text { where } B_{\text {scales, } \mathrm{e}}=0 .
\end{array}\right.
$$

A morphological reconstruction on the inverse data set, as given by

$$
I_{\mathrm{rec}}=-\left(\left(-I_{0.3}\right) \operatorname{rec}_{\oplus 8}\left(-I_{\text {marker }}\right)\right),
$$

then yields a surface where (possibly cracked) scales remain while cracks in the steel are filled in (fig. 5e). A filter identifying cavities located where trenches were found in the low resolution step (section III-B) can then be obtained by performing an opening by reconstruction on the filled in regions, using the trench marker $B_{\text {trenches }}$ (fig. 4d) from (3). This results in a binary filter

$$
B_{\text {cav }}=\left(I_{\text {rec }}>I_{0.3}\right) \operatorname{rec}_{\oplus 8} B_{\text {trenches }},
$$

where cavities in the steel surface, as opposed to scales, are identified (fig. 5f). This filter can then be used to exclude scales and other non-cavities from further crack analysis.

\section{Crack Signature Extraction (High Resolution)}

In non-scale regions where longitudinal cavities were found, a more detailed investigation is needed. Fig. 6a shows an example of data processed in this step, $I_{0.1}$.

1) Identifying sharp discontinuities: We restrict our attention to sharp discontinuities in the data in the horizontal (transversal) direction. This is done by retrieving a binary filter

$$
B_{\text {discont }}=\left\{\begin{array}{cl}
I_{0.1} \bullet l_{\mathrm{h}, 0.3 \mathrm{~mm}}>I_{0.1} & \text { where } B_{\text {cav }}=1, \\
0 & \text { where } B_{\text {cav }}=0,
\end{array}\right.
$$

where $l_{\mathrm{h}, 0.3 \mathrm{~mm}}$ is a horizontal line structuring element of length $0.3 \mathrm{~mm}$ (fig. 6b). $B_{\text {cav }}$ (fig. 5f) is the cavity filter obtained from (10).

2) Depth estimation: The depths $I_{\text {depth }}$ of horizontal discontinuities in the data are then approximated as

$$
I_{\text {depth }}=\left\{\begin{array}{cl}
I_{0.1} \bullet l_{\mathrm{h}, 5 \mathrm{~mm}}-I_{0.1} & \text { where } B_{\text {discont }}=0, \\
0 & \text { where } B_{\text {discont }}=1,
\end{array}\right.
$$

where $l_{\mathrm{h}, 5 \mathrm{~mm}}$ is a horizontal line structuring element of length $5 \mathrm{~mm}$. Hence, the crack depth is defined as the difference between the actual data value and the minimum height to which a horizontal line of length $5 \mathrm{~mm}$ can be pushed down into the data. The resulting image is shown in fig. $6 \mathrm{c}$.

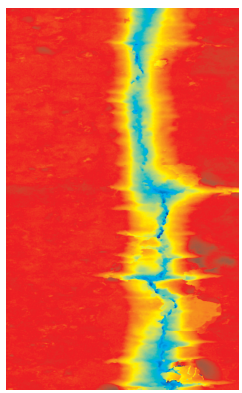

(a)

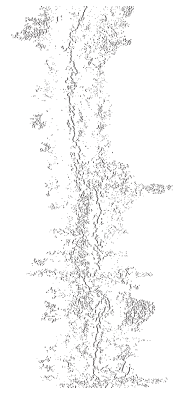

(b)

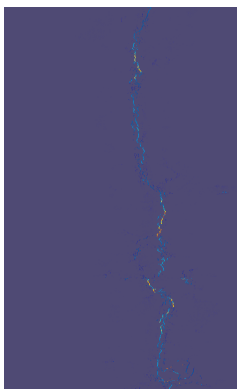

(c) (d)

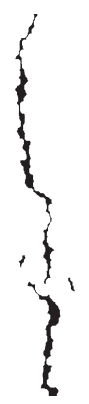

(e)

Fig. 6. The different images obtained during the crack signature extraction: $I_{0.1}$ (a), $B_{\text {discont }}(\mathrm{b}), I_{\text {depth }}$ (c), $B_{\text {cracks }}\left(\right.$ d), and $B_{\mathrm{L}, 10}$ (e).

3) Obtaining crack signatures: From the approximated crack depth $I_{\text {depth }}$, we define potential crack signatures $B_{\text {cracks }}$ as

$$
B_{\text {cracks }}=I_{\text {depth }}>0.1 \text {, }
$$

neglecting depths smaller than $0.1 \mathrm{~mm}$ (see fig. 6d).

4) Linking crack signatures: Neighboring remaining signatures are linked together while unconnected signatures are discarded, using a morphological filter given by the recursive expression

$B_{\mathrm{L}, k}=\left\{\begin{array}{cc}B_{\text {cracks }}, & k=0, \\ \left(B_{\mathrm{L}, k-1} \bullet d_{k}\right) \operatorname{rec}_{\oplus 8}\left(\left(B_{\mathrm{L}, k-1} \bullet d_{k}\right) \circ l_{k}\right), & k>0 .\end{array}\right.$

Here, $d_{k}$ is a disk structuring element of radius $k \mathrm{~mm}$ and $l_{k}$ a vertical line structuring element of length $2 k+0.1 \mathrm{~mm}$, where $k=\{0.1,0.2,0.3, \ldots, 1.0\}$. The filter is a modified close/open alternating filter. Instead of using the same element for both the closing and the opening a disk is used to close up parts that are not perfectly vertically aligned, while a vertical line is used to restrict the filter output to vertical features. An opening by reconstruction in each step makes the filter more conservative, keeping more of the grouped regions in each step. This linking produces a segmented data set, where each connected component marks a region corresponding to a potential crack in the steel surface (see fig. 6e).

5) Excluding small regions: After the linking procedure, small regions are discarded by an area opening of size $5 \mathrm{~mm}^{2}$. Connected components in the remaining signatures are reported as potential crack segments. 


\section{Classification}

Once the data has been segmented, shape features are extracted for each reported connected component. These parameters can then be used to classify the potential cracks as (true) cracks or non-cracks. The potential cracks reported by the segmentation was first manually classified, producing a reference set where each connected region is marked as being a crack, a non-crack or unclassified (ambiguous regions where cracks are likely although no open crack is visible). This classification was based upon resemblance to the identified cracks in the model set (see section II).

\section{A. Variable Extraction and Orientation Thresholding}

The depth of each connected region is reflected by its median depth, retrieved from (12). Since the region obtained from the morphological filtering is in general wider than the actual thin crack, we only consider pixels where the estimated depth is non-zero. Other shape parameters are obtained by approximating each connected region by an ellipse defined by the same corresponding second central moments (see fig. 7). The lengths of the minor and major axes can then be considered to reflect the length and width of the crack, and the orientation is defined by the minimum absolute angle between the major axis and the $\mathrm{x}$-axis (the transversal direction).

We exclude all connected regions with orientation less than $45^{\circ}$ from further analysis, since these can hardly be considered longitudinal. Box plots for the obtained variable distributions for set A within the manually defined groups cracks and noncracks are presented in fig. 8 .

1) Orientation: The orientation box plot in fig. 8a shows that the identified cracks indeed show a longitudinal behavior, and the orientation threshold could probably be raised. In this work however, the number of available samples was already considered scarce enough from a statistical point of view.

2) Minor axis: In fig. 8b, we see that cracks in the model set are in general wider than other reported regions. This is considered to be a result of frequently occurring zig-zag patterns in the cracks in set A - a property we can hardly assume to be true in general. Thus, the minor axis is considered unsuitable for classification.

3) Major axis \& median depth: The major axis and the median depth (figs. 8c and 8d) show separation between the groups, indicating that the segmentation succeeds in finding

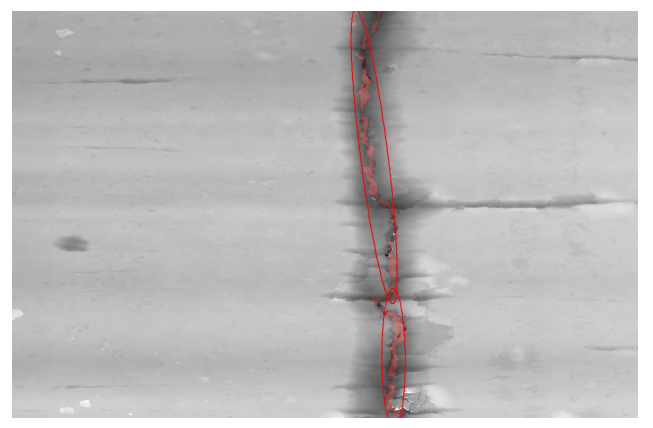

Fig. 7. Ellipses fitted to the connected regions reported by the segmentation.

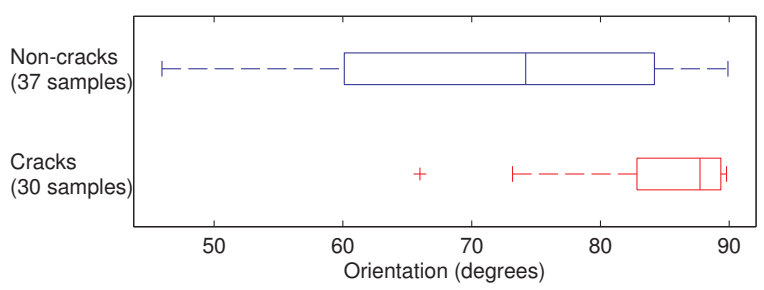

(a)

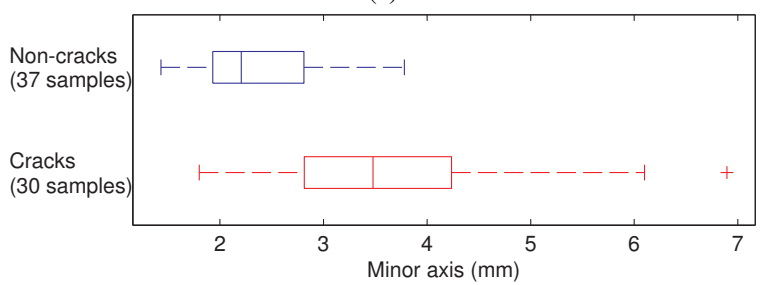

(b)

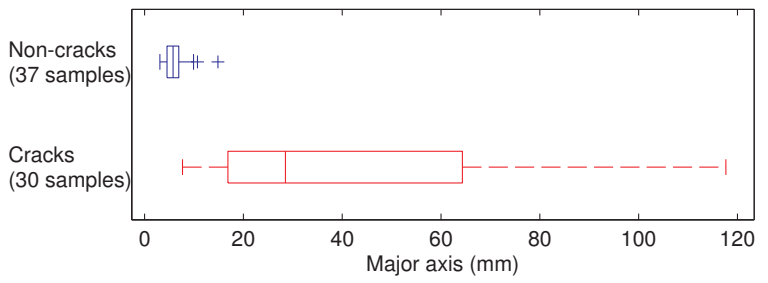

(c)

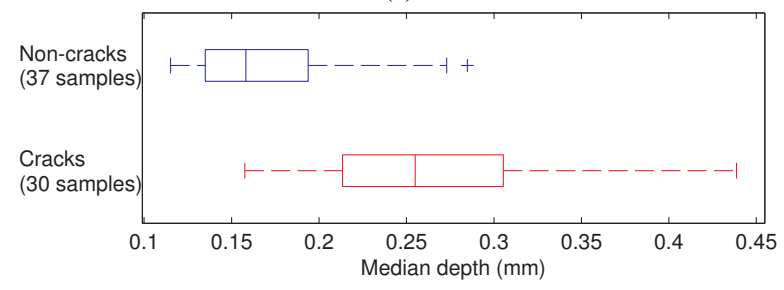

(d)

Fig. 8. Box plots for studied variables in the model set; orientation (a), minor axis length (b), major axis length, (c), and median depth (d), respectively.

long, deep regions where cracks have been manually identified. These variables are therefore considered suitable for classification of the crack signatures.

\section{B. Logistic Regression}

In fig. 9a, the manually classified regions are marked in the space spanned by the two variables selected for classification; the major axis and the median depth. While the number of regions containing cracks is not very high, we see that there is indeed a tendency for crack regions to be longer and deeper than the non-crack noise from the segmentation. In this space, a statistical classifier was then obtained by fitting a logistic regression model to the manually classified groups containing cracks and non-cracks. This method was chosen because it does not assume normally distributed data [32], [33], which makes it a suitable choice for the available model data where crack samples are quite sparsely distributed within the upper right part of fig. 9a.

1) Posterior probability: Logistic regression can be used to estimate the posterior probability for each sample to belong to a certain group. More specifically, an $n$-variable logistic regression model is defined by the parameters $\left\{\beta_{0}, \beta_{1}, \beta_{2}, \ldots, \beta_{n}\right\}$, which for each sample represented by the 


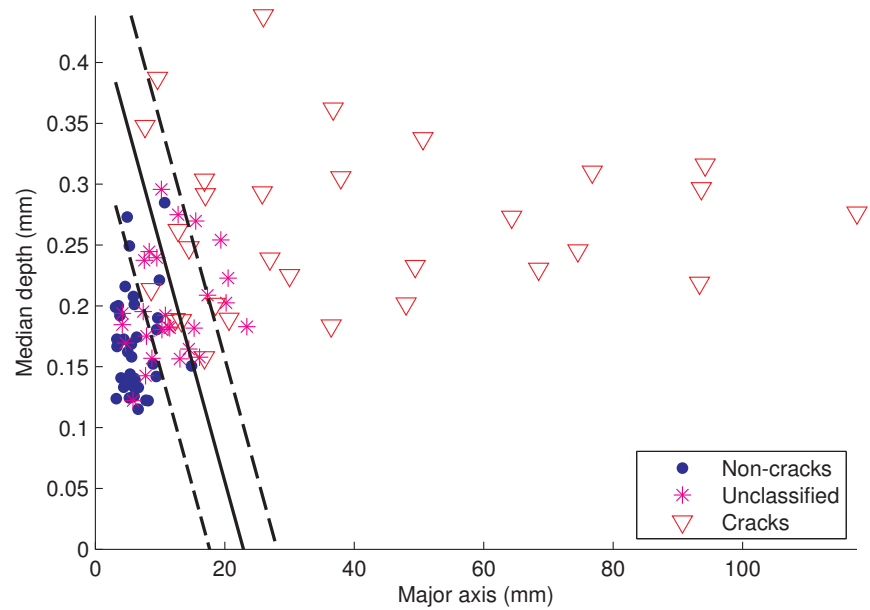

(a)

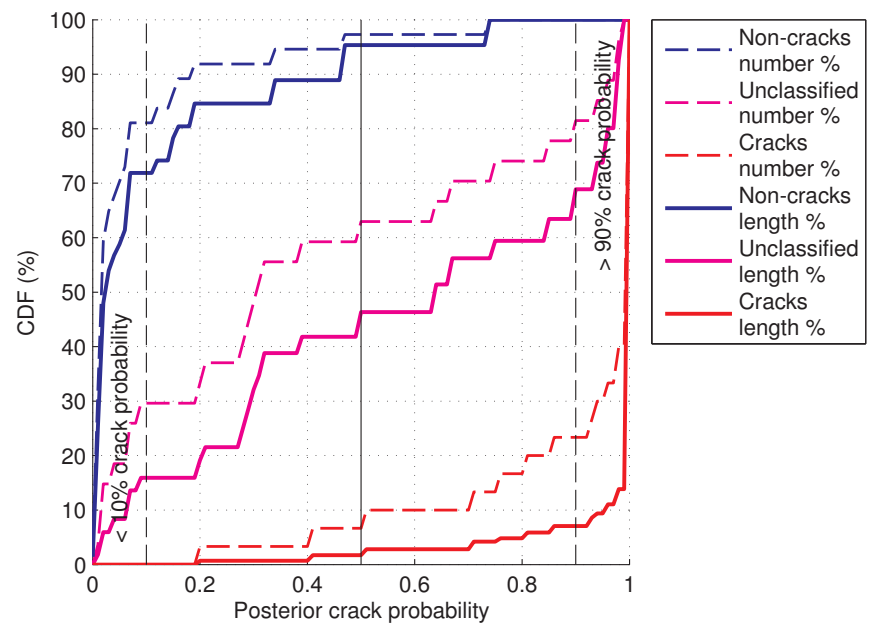

(b)

Fig. 9. The two-dimensional classification space (a) and the corresponding CDFs (b). In both figures, lines marking 10\%, 50\% and 90\% crack probabilities (seen from left to right) are displayed.

variables $\left\{x_{1}, x_{2}, \ldots, x_{n}\right\}$ yields a posterior probability given by

$$
P_{\text {posterior }}=\frac{1}{1+e^{-\left(\beta_{0}+\sum_{k=1}^{k=n} \beta_{k} x_{k}\right)}} .
$$

The interested reader is referred to Afifi [33] and Dobson [34] for more details on the topic of logistic regression.

2) Probability threshold: Cumulative distribution functions (CDFs) representing the posterior probabilities, given for each classified region by (15), are presented in fig. 9b. The solid lines represents the CDFs in percentage of the total number of regions for each manually classified group, while the dashed lines represents the CDFs in total length percentage (total detected length of cracks vs. total length of manually marked cracks) for each manually classified group. A probability percentage threshold, which corresponds to a boundary line in fig. 9a, can be used to identify cracks in the data. It should be noted that the classifier is blind to what the segmentation fails to report. Also, as mentioned, regions with orientation less than $45^{\circ}$ have already been discarded at this stage.

By selecting a probability value for thresholding higher than $50 \%$ we can avoid the misclassification risk near the
$50 \%$ boundary, which will make the system more robust. This threshold should be a trade-off between $100 \%$ crack detection and $0 \%$ false positives. Since our primary focus in this work is on the latter, we want a high threshold value which still classifies most identified cracks correctly. Fig. 9b shows that we can set a probability requirement as high as $90 \%$ and still keep $93 \%$ of the total length of connected regions containing manually identified cracks, while safely avoiding any false positives. However, the unclassified samples must be taken into account as well. We can get worst-case scenarios by considering the two extremes;

1) all unclassified regions are assumed to be non-cracks, and

2) all unclassified regions are assumed to be cracks.

In case 1 , the false detection rate is $21 \%$ of the total noncrack length, or $116 \mathrm{~mm}$ falsely reported cracks. In case 2, on the other hand, $81 \%$ of the total crack length is correctly classified. The truth should lie in-between these two extremes. The $21 \%$ false detection rate given by case 1 should be quite pessimistic, since the vast majority of the unclassified regions are located in the proximity of manually identified cracks, but cannot be ruled out at this point.

\section{RESUlTS}

The performance of the presented method was validated by segmenting and classifying a second independent validation set $\mathrm{B}$, containing 323 regions of $3 \mathrm{D}$ surface data collected from four different steel slabs.

\section{A. Segmentation Results}

The connected regions retrieved from the segmentation included $82 \%$ of the total length of the manually marked cracks. Correct crack signatures were extracted from all 17 images manually marked as containing cracks.

Descriptive shape parameters were then extracted from each detected region. Box plots are presented in fig. 10, together with the previous results for set A for comparison. As before, all regions with less than $45^{\circ}$ absolute orientation relative to the $\mathrm{x}$-axis have at this stage been discarded. Variable distributions for set $\mathrm{B}$ validate the earlier observations for set $\mathrm{A}$ (section IV-A). In particular, the problem with using the minor axis for classification is evident - crack widths in set B are in general smaller than in set $\mathrm{A}$, and are to a large extent overlapping the widths of the non-crack regions.

\section{B. Classification Results}

The extracted variables were used as input to the logistic regression model obtained from set $\mathrm{A}$, and the resulting classification was compared to a manual classification. Results are presented in fig. 11. In fig. 11a, two dashed lines marking $10 \%$ and $90 \%$ crack probability, respectively, as well as a solid line representing the $50 \%$ probability boundary are shown in the $2 \mathrm{D}$-space spanned by the major axis and the median depth. Fig. 11b shows the corresponding cumulative distribution functions (CDFs) for the region-wise posterior probabilities, presented in both number of regions and length percentage. 


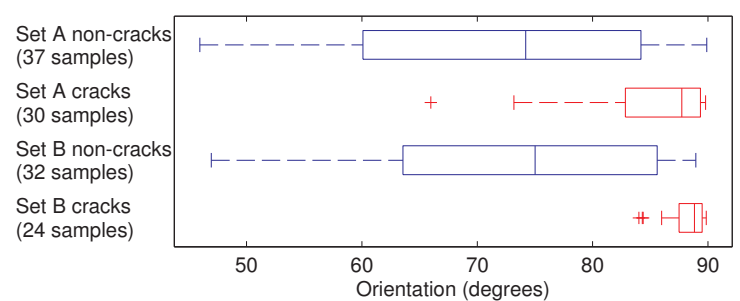

(a)

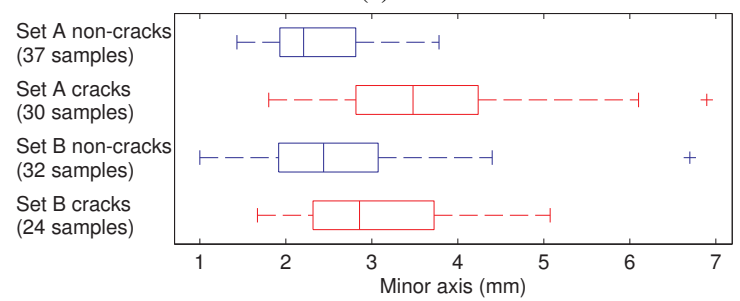

(b)

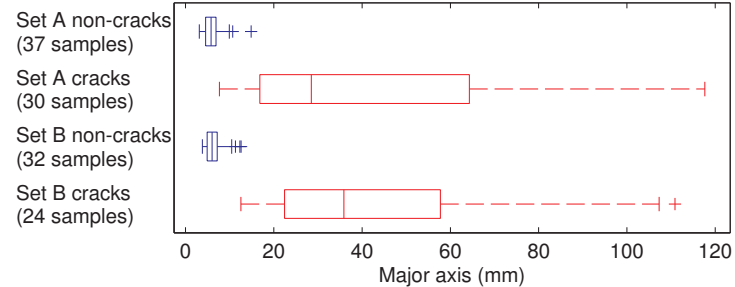

(c)

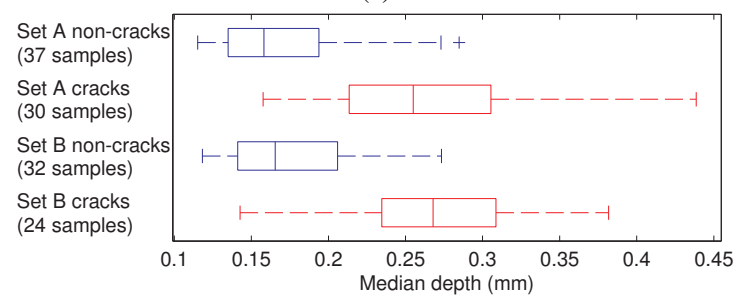

(d)

Fig. 10. Box plots for studied variables; orientation (a), minor axis length (b), major axis length, (c), and median depth (d), respectively. Results for set $\mathrm{A}$ are presented again for comparison.

By comparing CDFs for crack and non-crack regions the separation tendency for the two groups can be evaluated. Probabilities are obtained from the logistic regression model, and are thereby based on the model data.

It is clear that at the $90 \%$ crack probability level, suggested in section IV, the classification of the validation set is as accurate as for the model set. $83 \%$ of the number of segmented crack regions, or $94 \%$ of the total length of segments containing cracks, are correctly classified. Still, no false positives are reported for the manually classified non-cracks on that probability level. Taking the unclassified regions into account by evaluating worst-case scenarios, as described previously in section IV-B under Probability threshold, results in less than $23 \%$ false positives $(131 \mathrm{~mm})$ and at least $83 \%$ correcly classified cracks (with respect to total length). Here, as well as for the model set, only 2 regions containing cracks are completely missed. As for the model set, the missed regions contain only short cracks.

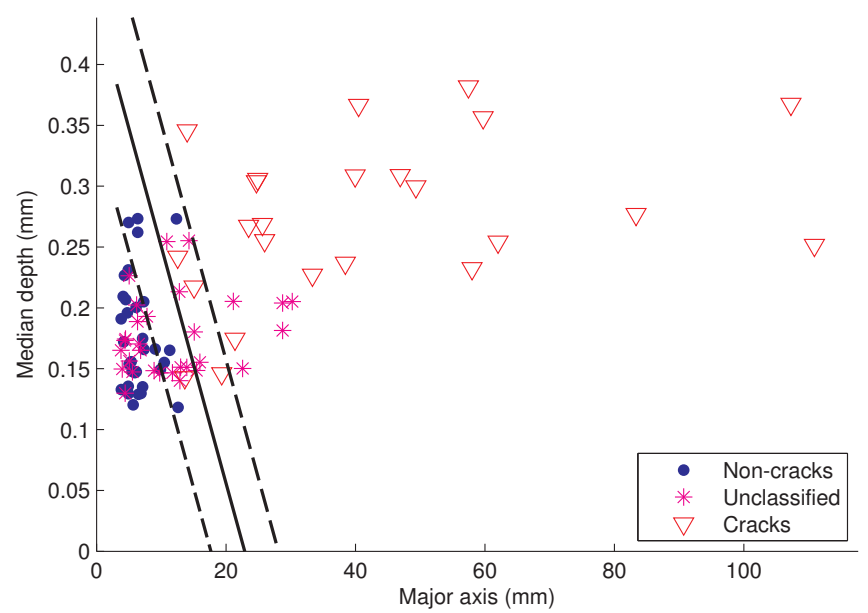

(a)

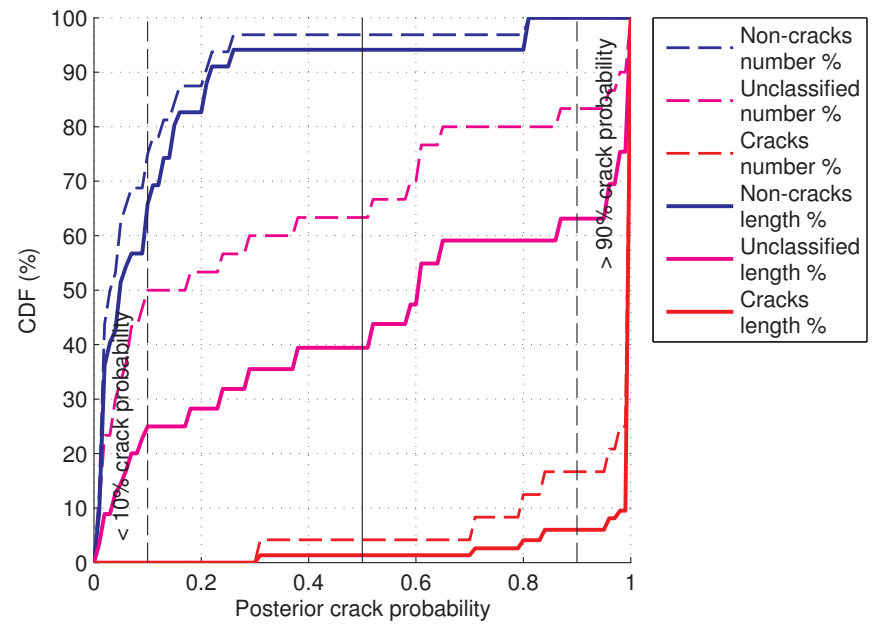

(b)

Fig. 11. Classification of the validation data (set B), in the two-dimensional classification space (a) and in CDF-format (b). In both figures, lines marking $10 \%, 50 \%$ and $90 \%$ crack probabilities (seen from left to right) are displayed.

\section{Combined Results}

The segmentation successfully reports $527 / 645 \mathrm{~mm}(82 \%)$ of the manually marked cracks, while the classification identifies $490 / 528 \mathrm{~mm}(93 \%)$ of these segmented regions as cracks. This evaluation of the classification differs from the $94 \%$ stated in the previous section, since we do not here consider parts of the linked connected regions that do not overlap the manually marked visible crack segments. In total, $490 \mathrm{~mm}$ of the total $645 \mathrm{~mm}$ manually marked crack length were correctly classified, yielding a success rate (in length percentage) of $76 \%$. The corresponding number for the model data is $73 \%$.

Worst-case scenario evaluation of the complete system is less trivial. A reference for non-crack and unclassified regions is hard to retrieve, since these regions result from the segmentation and cannot be manually marked before that point. What we can say, however, is that less than $131 \mathrm{~mm}$ crack length is falsely identified. This number can be compared to the total $30600 \mathrm{~mm}$ long sequence of $150 \mathrm{~mm}$ wide sections of data present in the validation data set. 


\section{DISCUSSION}

While the amount of collected 3D data could certainly be improved in a continued future study, the results show clear tendencies for the available surface profiles:

- The presented segmentation algorithm successfully extracts more than $80 \%$ of the total crack length present in the data, while discarding most potential pseudo-defects (non-defect surface features similar to defects).

- At a $90 \%$ probability level, the statistical classification individually has a crack detection accuracy of over $90 \%$ with respect to the total manually identified crack length, while discarding all remaining manually identified pseudo-defects. Taking ambiguous, unclassified regions into account gives a worst-case detection rate of over $80 \%$ and a worst-case false detection rate of $23 \%$ (corresponding to a length of $131 \mathrm{~mm}$ ).

- The combined system (segmentation and classification) detection success rate, with respect to the total length of manually marked distinct open cracks, is over $70 \%$.

- Only a few of the regions containing cracks are completely missed (a crack region may be identified even though all crack length within the region is not detected) and, most importantly,

- no false positives are reported within the manually classified data and false detection in total is less than $131 \mathrm{~mm}$ among the $30600 \mathrm{~mm}$ long sequence of $150 \mathrm{~mm}$ wide regions the validation set.

These numbers indicate that cracks to a large extent can be separated from non-crack data, and an accurate automated crack-detection system based on the presented method is feasible. Some cracks - predominantly smaller ones - will likely remain undetected as a result of the trade-off between $100 \%$ detection and $0 \%$ false positives, but longer cracks can be quite safely identified. It is therefore important to link crack segments together whenever possible, so that they get long enough to be classified correctly. A potential method for achieving this is the Hough transform, which has not been used in this work.

The crack probability measure allows for a more continuous crack resemblance assessment for each region, rather than a strict binary classification. This property can be used to avoid false positives. By thresholding at a higher crack probability value, false positives can be avoided at the cost of successful classification of true cracks. Tuning this threshold with respect to associated costs can then be considered as an optimization problem.

The presented method relies on a number of parameters, which are set experimentally (and with respect to the actual physical measures they represent). A completely adaptive parameter selection would hardly be useful, due to the underlying physical quantities the numbers represent, but it may be possible to set them more systematically. In particular, the impact on these parameters from different types of steel grades should be further investigated. It may also be possible to reduce the number of parameters by introducing other methods such as watershed segmentation or the Hough transform, but this lies beyond the scope of this work.

\section{CONCLUSION}

The presented system provides a crack probability measure for each detected potential crack. We have shown that the data can be classified at $90 \%$ crack probability, resulting in less than $131 \mathrm{~mm}$ of the $30600 \mathrm{~mm}$ long sequence of $150 \mathrm{~mm}$ wide regions in the validation set being detected as cracks without being manually identified as such. At this crack probability level, over $70 \%$ of the manually identified crack length was still successfully detected. No cracks were falsely detected in regions where manual identification completely ruled out the existence of cracks.

More data would allow for a more thorough statistical investigation of potential classification variables as well as a more accurate crack probability estimation, but the presented results clearly show the potential of the method. This work thereby provides a start for further development of the system into a fully automated morphology-based on-line crack detection installation.

\section{FUTURE WORK}

The algorithm generally succeeds in identifying relatively long connected components where cracks are present. This makes the length an important variable in the classification, and cracks should therefore be linked together as much as possible so that they can be distinguished from noise. For this purpose, use of the Hough transform should be investigated. Combining data in several images, thereby providing more high level knowledge, would also allow for longer estimated crack lengths and thereby even more extreme major axes for regions representing very long cracks.

In addition to the Hough transform, watershed segmentation should also be considered in a future study - with focus on seed selection and how over-segmentation can be effectively reduced.

The handling of missing data, by setting such pixels to the minimum value of the $150 \times 100 \mathrm{~mm}$ data being processed, did not effect classification results but is quite crude and could certainly be improved. The authors have previously considered reconstruction of occluded regions in 3D profile data of rocks [35], but such methods are usually quite computationally demanding and thereby hard to implement in an online system. A more locally adapted reconstruction of missing data should make the segmentation more robust though, and could be worth looking into further.

Finally, more extensive measurements on different types of steel would provide more statistical data and allow for a more thorough investigation of the relation between steel grades and the parameters used, thereby resulting in a more accurate crack probability estimation.

\section{ACKNOWLEDGMENT}

The authors would like to thank ProcessIT Innovations for all their invested time and effort. We also thank SSAB for their participation and support. Finally, we thank our measurement technology partners, Kemi Technical University of Applied Sciences (KTUAS). In addition we acknowledge the EU INTERREG IVA Nord program for partially supporting this research. 


\section{REFERENCES}

[1] "World steel in figures," World Steel Association, Brussels, Belgium, Tech. Rep., 2011. [Online]. Available: www.worldsteel.org

[2] R. Mahapatra, J. Brimacombe, I. Samarasekera, N. Walker, E. Paterson, and J. Young, "Mold behavior and its influence on quality in the continuous casting of steel slabs: Part i. industrial trials, mold temperature measurements, and mathematical modeling," Metallurgical and Materials Transactions B, vol. 22, pp. 861-874, 1991, 10.1007/BF02651163.

[3] X. Li, S. Tso, X. Guan, and Q. Huang, "Improving automatic detection of defects in castings by applying wavelet technique," IEEE Transactions on Industrial Electronics, vol. 53, no. 6, pp. 1927-1934, 2006.

[4] J. Yun, S. Choi, B. Seo, C. Park, and S. Kim, "Defects Detection of Billet Surface Using Optimized Gabor Filters," in Proceedings of the 17th IFAC World Congress. The International Federation of Automatic Control, 2008.

[5] D. Lee, Y. Kang, C. Park, and S. Won, "Defect Detection Algorithm in Steel Billets using Morphological Top-Hat Filter," in IFAC Workshop on Automation in Mining, Mineral and Metal Industry (IFACMMM), 2009.

[6] R. T. Chin and C. A. Harlow, "Automated visual inspection: A survey," IEEE Transactions on Pattern Analysis and Machine Intelligence, vol. PAMI-4, no. 6, pp. 557-573, nov. 1982.

[7] R. Chin, "Automated visual inspection: 1981 to 1987," Computer Vision, Graphics, and Image Processing, vol. 41, no. 3, pp. 346-381, 1988.

[8] E. Bayro-Corrochano, "Review of automated visual inspection 19831993, Part I: conventional approaches," in Proceedings of SPIE, vol. 2055, 1993, p. 128.

[9] — - "Review of automated visual inspection 1983-1993, Part II: approaches to intelligent systems," in Proceedings of SPIE, vol. 2055, 1993, p. 159.

[10] T. Newman and A. Jain, "A survey of automated visual inspection," Computer vision and image understanding, vol. 61, no. 2, pp. 231-262, 1995.

[11] E. Malamas, E. Petrakis, M. Zervakis, L. Petit, and J. Legat, "A survey on industrial vision systems, applications and tools," Image and Vision Computing, vol. 21, no. 2, pp. 171-188, 2003.

[12] J. Yun, S. Choi, Y. Jeon, D. Choi, and S. Kim, "Detection of line defects in steel billets using undecimated wavelet transform," in International Conference on Control, Automation and Systems, 2008. IEEE, 2008, pp. $1725-1728$.

[13] Y. Jeon, J. Yun, D. Choi, and S. Kim, "Defect detection algorithm for corner cracks in steel billet using discrete wavelet transform," in ICCASSICE, 2009. IEEE, 2009, pp. 2769-2773

[14] M. Yazdchi, A. Mahyari, and A. Nazeri, "Detection and classification of surface defects of cold rolling mill steel using morphology and neural network," in International Conference on Computational Intelligence for Modelling Control \& Automation, 2008. IEEE, 2008, pp. 1071-1076.

[15] F. Pernkopf, "3D surface acquisition and reconstruction for inspection of raw steel products," Computers in Industry, vol. 56, no. 8-9, pp. 876$885,2005$.

[16] F. Pernkopf and P. O'Leary, "Image acquisition techniques for automatic visual inspection of metallic surfaces," NDT \& E International, vol. 36, no. 8, pp. 609-617, 2003

[17] I. Alvarez, J. Marina, J. Enguita, C. Fraga, and R. Garcia, "Industrial online surface defects detection in continuous casting hot slabs," in Proceedings of SPIE, vol. 7389, 2009, p. 73891X.

[18] B. Thomas, "On-line Detection of Quality Problems in Continuous Casting of Steel," in Proceedings of the International Symposium on Process Control and Optimization in Ferrous and Nonferrous Industry, TMS, Warrendale, PA,(Chicago, IL), 2003.

[19] P. Meilland, "Novel Multiplexed Eddy-Current Array for Surface Crack Detection on Rough Steel Surface," Proc. 9th ECNDT, Berlin, 2006.

[20] T. Nishimine, O. Tsuyama, T. Tanaka, and H. Fujiwara, "Automatic magnetic particle testing system for square billets," in Conference Record of the 1995 IEEE Industry Applications Conference, vol. 2. IEEE, 2002, pp. 1585-1590.

[21] M. Allazadeh, C. Garcia, K. Alderson, and A. Deardo, "Ultrasonic image analysis of steel slabs," Advanced materials \& processes, vol. 166 , no. 12 , pp. 26-27, 2008.

[22] J. Sirgo, R. Campo, A. Lopez, A. Diaz, and L. Sancho, "Measurement of centerline segregation in steel slabs," in Conference Record of the 2006 IEEE Industry Applications Conference, vol. 1. IEEE, 2006, pp. 516-520.

[23] V. Rathod and R. Anand, "A comparative study of different segmentation techniques for detection of flaws in nde weld images," Journal of Nondestructive Evaluation, vol. 31, pp. 1-16, 2012.
[24] S. Nashat, A. Abdullah, and M. Abdullah, "A robust crack detection method for non-uniform distributions of coloured and textured image," in 2011 IEEE International Conference on Imaging Systems and Techniques (IST), may 2011, pp. 98-103.

[25] S. Jiaxin, D. Dong, Z. Xinjie, and W. Li, "Weld slim line defects extraction based on adaptive local threshold and modified hough transform," in 2nd International Congress on Image and Signal Processing, 2009. CISP '09., oct. 2009, pp. 1-5.

[26] J. Angulo and D. Jeulin, "Stochastic watershed segmentation," in Proceedings of ISMM, 8th International Symposium on Mathematical Morphology, 2007.

[27] T. T. Vu, F. Yamazaki, and M. Matsuoka, "Multi-scale solution for building extraction from lidar and image data," International Journal of Applied Earth Observation and Geoinformation, vol. 11, no. 4, pp. $281-289,2009$

[28] J. Lee, M. Smith, L. Smith, and P. Midha, "A mathematical morphology approach to image based 3D particle shape analysis," Machine Vision and Applications, vol. 16, no. 5, pp. 282-288, 2005.

[29] M. Thurley, "Automated online measurement of limestone particle size distributions using 3D range data," Journal of Process Control, vol. 21 , no. 2, pp. 254-262, 2011.

[30] M. Thurley and T. Andersson, "An industrial 3D vision system for size measurement of iron ore green pellets using morphological image segmentation," Minerals Engineering, vol. 21, no. 5, pp. 405-415, 2008.

[31] E. Dougherty and R. Lotufo, Hands-on Morphological Image Processing, ser. Tutorial Texts in Optical Engineering. Bellingham, Washington, USA: SPIE - The International Society for Optical Engineering, 2003, vol. TT59.

[32] D. Johnson, Applied Multivariate Methods for Data Analysts. Pacific Grove, California, USA: Duxbury Press, 1998.

[33] A. Afifi, V. Clark, and S. May, Computer-aided multivariate analysis, 4th ed. Boca Raton, Florida, USA: CRC, 2004.

[34] A. Dobson, An Introduction to Generalized Linear Models, 2nd ed. Boca Raton, Florida, USA: CRC, 2002.

[35] A. Landström, F. Nellros, H. Jonsson, and M. Thurley, "Image reconstruction by prioritized incremental normalized convolution," in Image Analysis, ser. Lecture Notes in Computer Science, A. Heyden and F. Kahl, Eds. Springer Berlin / Heidelberg, 2011, vol. 6688, pp. 176185.

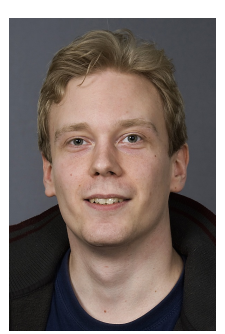

Anders Landström received the M.S. degree within engineering physics at Luleå University of Technology, Sweden, in 2010, and is currently working towards a Ph.D. degree within signal processing at Luleå University of Technology, Sweden.

The focus of his research is on applying image analysis to practical problems, providing automated solutions for use within industrial processes.

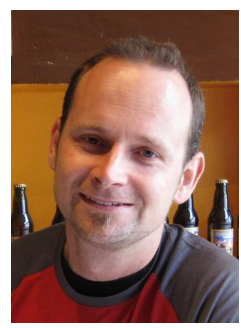

Matthew Thurley is associate professor in image analysis and industrial machine vision and is founder of the industrial image analysis group at Lulea University of Technology, Sweden. He has a strong focus on machine vision research to provide fully automated online measurement solutions to industrial problems particularly within the mining and steel industries. Specifically within surface inspection in steel, quantitative analysis of microscopy structure and particle size measurement of piled particulate material in mining. Matthew's research is on industrial measurement projects with image analysis, geometric algorithms, mathematical morphology, and classification. 